\title{
Rate and rhythm control showed similar symptom improvement in atrial fibrillation
}

\author{
Hohnloser SH, Kuck KH, Lilienthal J, for the PIAF Investigators. Rhythm or rate control in atrial fibrillation- \\ pharmacological intervention in atrial fibrillation (PIAF): a randomised trial. Lancet 2000 Nov 25;356:1 789-94.
}

\section{QUESTION: In patients with atrial fibrillation (AF), how do rate control and sinus rhythm control compare in improving symptoms?}

\section{Design}

Randomised (unclear allocation concealment*), unblinded,* controlled trial with 12 months follow up.

\section{Setting}

21 centres in Germany.

\section{Patients}

252 patients who were $18-75$ years of age (mean age $60 \mathrm{y}$, $73 \%$ men) and who presented with symptomatic persistent $\mathrm{AF}$ between 7 and 360 days in duration. Exclusion criteria included congestive heart failure, New York Heart Association class IV; unstable angina; acute myocardial infarction in the previous 30 days; $\mathrm{AF}$ with mean rate $<50$ beats/minute; AF in conjunction with the Wolff Parkinson White syndrome; and coronary artery bypass graft or valve replacement in the previous 3 months. Follow up was $96 \%$.

\section{Intervention}

Patients were allocated to rate control $(n=125)$ or to rhythm control $(n=127)$. In rate control, the therapeutic goal was to achieve an improvement in symptoms by controlling ventricular rate with the use of diltiazem, $90 \mathrm{mg}$, 2-3 times/day. In rhythm control, the aim was to prevent recurrent $\mathrm{AF}$ with the use of amiodarone, $600 \mathrm{mg}$ /day for 3 weeks, and electrical cardioversion thereafter if sinus rhythm was not restored. Amiodarone, $200 \mathrm{mg}$ /day, was given for maintenance of sinus rhythm.

\section{Main outcome measures}

Improvement in symptoms (elimination of palpitations and reduction of dyspnoea and dizziness). Secondary outcomes were easy fatigability (6 minute walking test), change in mean heart rate, hospital admission, and quality of life.

\section{Main results}

The study had $90 \%$ power to detect a $50 \%$ to $70 \%$ difference in symptom improvement. The groups did not differ for symptom improvement: at 12 months, $56 \%$ of rate control patients improved compared with $60 \%$ of rhythm control patients $(\mathrm{p}>0.2)$. Patients in the rhythm control group achieved a greater walking distance in 6 minutes than patients in the rate control group $(\mathrm{p}=0.008$ at 12 mo). Heart rate decreased in both groups, from 88 to 81 beats/minute in the rate control group $(p=0.001)$ and from 86 to 78 beats/minute in the rhythm control group $(p=0.002)$. Fewer patients in the rate control group, than in the rhythm control group, were admitted to hospital at least once $(24 \% v 69 \%, \mathrm{p}=0.001) .68 \%$ of admissions in rate control patients were for drug related adverse effects; $67 \%$ of admissions in rhythm control patients were for electrical cardioversion, and 27\% were for amiodarone related adverse effects. The groups did not differ for quality of life measures.

\section{Conclusion}

In patients with atrial fibrillation, rate control and sinus rhythm control did not differ for symptom improvement.

*See glossary.

\section{COMMENTARY}

It is unclear whether AF is better treated by suppression of tachycardia with diltiazem or by cardioversion and maintenance of sinus rhythm with amiodarone. ${ }^{1}$ The unblinded randomised trial by Hohnloser et al confirmed conventional opinion that rhythm control provides better exercise tolerance than rate control. This benefit came at a cost: more hospital admissions for electrical cardioversion or drug toxicity in the rhythm control group.

Despite improved exercise tolerance in the amiodarone group, the groups did not differ for symptoms (palpitations, dyspnoea, or dizziness) or quality of life. Equivalence of subjective outcomes may represent therapeutic equivalence, or it may be a result of subtle effects of the acknowledged bias of the investigators against rhythm control. Alternatively, bias might explain the findings: 5 rate control patients had catheter induced atrioventricular node modification to optimise rate control, and second line management was left to the discretion of the investigators at each site rather than being explicitly included in the protocol.

The applicability of this study to practice is limited because most clinicians would not follow either of these simple strategies. Patients who failed amiodarone would probably be treated with repeated electrical cardioversion or other anti-arrhythmic drugs, such as procainamide. ${ }^{2}$ Patients who failed alternative anti-arrhythmics would probably be treated with rate control or with an alternative procedure, such as ablation of the atrioventricular node and a permanent pacemaker. ${ }^{3}$ The study design also fails to reflect clinicians' use of pathophysiological models of AF: the longer a patient is in AF, the more difficult it is to restore sinus rhythm. Most clinicians would more aggressively seek to restore sinus rhythm when the heart is apparently structurally normal than when mitral valve disease or atrial dilatation exists.

Two other studies are currently in progress (Atrial Fibrillation Follow up Investigation of Rhythm Management [AFFIRM] and Rate Control v Electrical Cardioversion [RACE]). These may provide additional guidance on the relative merits of tachycardia suppression or cardioversion in sinus rhythm.

Steven Belknap, MD
University of Illinois College of Medicine at Peoria
Peoria, Illinois, USA

1 Olgin JE, Zipes DP. Specific arrhythmias: diagnosis and treatment. In: Braunwald E, Zipes DP, Libby P, editors. Heart disease. 6th ed. Philadelphia: WB Saunders, 2001.

2 Falk RH. Atrial fibrillation. N Engl J Med 2001;344:1067-78.

3 Ozcan C, Jahangir A, Friedman PA, et al. Long-term survival after ablation of the atrioventricular node and implantation of a permanent pacemaker in patients with atrial fibrillation. N Engl J Med 2001;344:1043-51.
Sources of funding: Sanofil Synthelabo Research and Parke Davis Research.

For correspondence: Dr S H Hohnloser, Department of Medicine, Division of Cardiology, J W Goethe University,

Theodor-Stern-Kai 7, 60590 Frankfurt, Germany. 